\title{
Improved Force Field Model for the Deep Eutectic Solvent Ethaline: \\ Reliable Physicochemical Properties
}

Elisabete S. C. Ferreira ${ }^{1,2, *}$, Iuliia V. Voroshylova ${ }^{1,2}$, Carlos M. Pereira ${ }^{2, *}$, M. Natália D. S.

${ }^{1}$ Departamento de Química e Bioquímica, LAQV@REQUIMTE, Faculdade de Ciências, Universidade do Porto, Rua do Campo Alegre, 4169-007 Porto, Portugal

${ }^{2}$ Departamento de Química e Bioquímica, CIQ(UP), Faculdade de Ciências da Universidade do Porto, Rua do Campo Alegre, 4169-007 Porto, Portugal

*Email: elisabete.ferreira@fc.up.pt; cmpereir@fc.up.pt; $\underline{\text { ncordeir@fc.up.pt }}$

\section{Contents}

FFM1 and FFM2

FFM3

FFM4

FFM5

\section{FFM1 and FFM2}

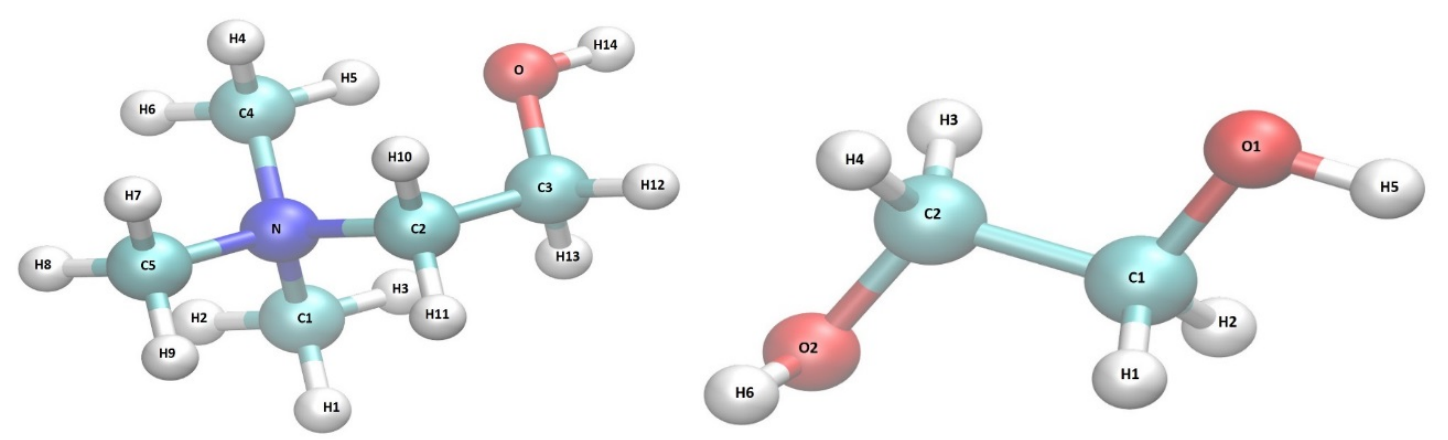

Figure S1. Nomenclature of atoms for choline $\left(\mathrm{Ch}^{+}\right)$and ethylene glycol (EG) for FFM1 and FFM2 


\section{FFM1:}

$\mathrm{Ch}^{+}$charges: J. Chem. Theory Comput. 5 (2009) 1038 - 1050, Parameters of OPLS-AA; EG and $\mathbf{C l}^{-}$charges and parameters: OPLS-AA.

\section{FFM2:}

$\mathbf{C h}^{+}$charges: J. Phys.Chem. B 117 ( 2013) 10250 - 10260;

EG and $\mathbf{C l}^{-}$charges and parameters: OPLS-AA

Table S1. Partial charges and nonbonded parameters used in FFM1 and FFM2 for choline $\left(\mathrm{Ch}^{+}\right)$.

\begin{tabular}{|c|c|c|c|c|c|}
\hline \multirow{2}{*}{ Atom name } & \multirow{2}{*}{$\begin{array}{l}\text { Atom } \\
\text { type }\end{array}$} & \multicolumn{2}{|c|}{ Partial charges for $\pm 1 \mathrm{e}$} & \multirow{2}{*}{$\begin{array}{l}\text { vdW radius } \\
\text { (nm) }\end{array}$} & \multirow{2}{*}{$\begin{array}{l}\text { Well depth (kJ } \\
\left.\mathrm{mol}^{-1}\right)\end{array}$} \\
\hline & & FFM1 & FFM2 & & \\
\hline $\mathrm{N}$ & opls_288 & 0.1640 & 0.0502 & 0.325 & $7.11280 \mathrm{E}-01$ \\
\hline $\mathrm{O}$ & opls_154 & -0.6545 & -0.6189 & 0.312 & $7.11280 \mathrm{E}-01$ \\
\hline $\mathrm{C} 1=\mathrm{C} 4=\mathrm{C} 5$ & opls_135 & -0.3847 & -0.1342 & 0.350 & $2.76144 \mathrm{E}-01$ \\
\hline $\mathrm{C} 2$ & opls_136 & -0.1111 & -0.0322 & 0.350 & $2.76144 \mathrm{E}-01$ \\
\hline $\mathrm{C} 3$ & opls 157 & 0.2318 & 0.1501 & 0.350 & $2.76144 \mathrm{E}-01$ \\
\hline $\mathrm{H} 1$ - H9 & opls_140 & 0.1934 & 0.1193 & 0.250 & $1.25520 \mathrm{E}-01$ \\
\hline $\mathrm{H} 10=\mathrm{H} 11$ & opls_140 & 0.1251 & 0.1116 & 0.250 & $1.25520 \mathrm{E}-01$ \\
\hline $\mathrm{H} 12=\mathrm{H} 13$ & opls_140 & 0.0398 & 0.0510 & 0.250 & $1.25520 \mathrm{E}-01$ \\
\hline H14 & opls_155 & 0.4537 & 0.4545 & 0.000 & $0.00000 \mathrm{E}+00$ \\
\hline
\end{tabular}

Table S2. Partial charges and nonbonded parameters used in FFM1 and FFM2 for ethylene glycol (EG).

\begin{tabular}{|c|c|c|c|c|}
\hline $\begin{array}{c}\text { Atom } \\
\text { name }\end{array}$ & Atom type & $\begin{array}{c}\text { Partial charges for } \\
\pm 1 \mathrm{e}\end{array}$ & $\begin{array}{c}\text { vdW radius } \\
(\mathrm{nm})\end{array}$ & $\begin{array}{c}\text { Well depth }(\mathrm{kJ} \\
\left.\text { mol }^{-1}\right)\end{array}$ \\
\hline $\mathrm{C} 1=\mathrm{C} 2$ & opls_157 & 0.145 & 0.350 & $2.76144 \mathrm{E}-01$ \\
\hline O1 = O2 & opls_169 & -0.700 & 0.307 & $7.11280 \mathrm{E}-01$ \\
\hline H1 - H4 & opls_140 & 0.060 & 0.250 & $1.25520 \mathrm{E}-01$ \\
\hline H5 = H6 & opls_170 & 0.435 & 0.000 & $0.00000 \mathrm{E}+00$ \\
\hline
\end{tabular}

\section{FFM3}

$\mathbf{C h}^{+}$charges and parameters: J. Chem. Theory Comput. 5 (2009) 1038 - 1050;

$\mathrm{Cl}^{-}$charges and parameters: OPLS-AA

EG charges and parameters: J. Phys.Chem. B 115 ( 2011) 3013 - 3019. 

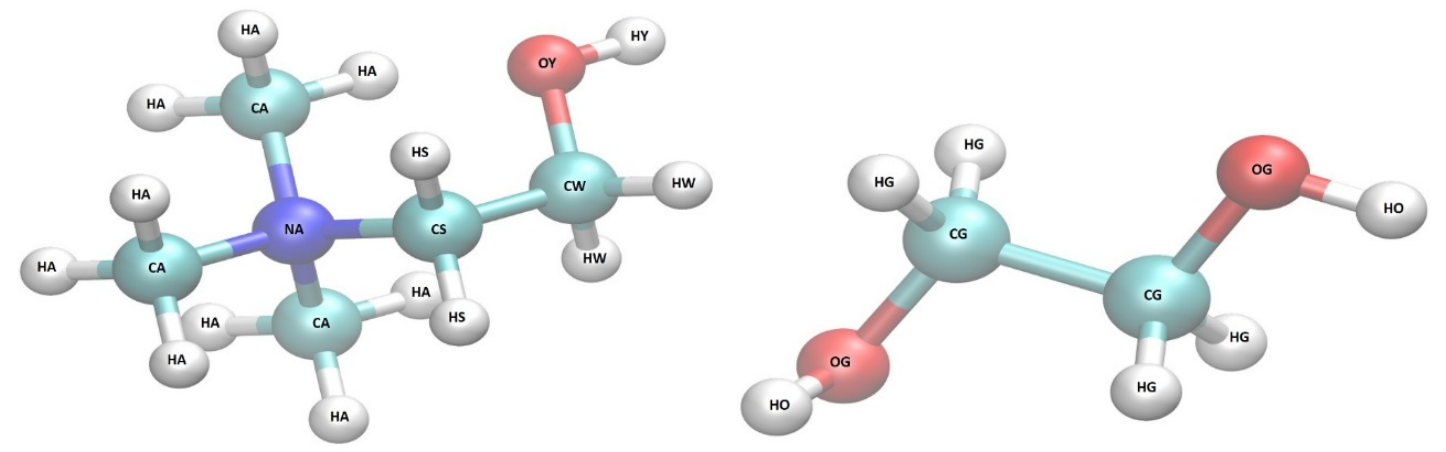

Figure S2. Nomenclature of atoms for choline $\left(\mathrm{Ch}^{+}\right)$and ethylene glycol (EG) for FFM3.

Table S3. Partial charges and nonbonded parameters used in FFM3 for choline $\left(\mathrm{Ch}^{+}\right)$and ethylene glycol (EG).

\begin{tabular}{|c|c|c|c|}
\hline Atom type & Partial charges for $\pm 1 \mathrm{e}$ & $\begin{array}{c}\text { vdW radius } \\
(\mathrm{nm})\end{array}$ & $\begin{array}{c}\text { Well depth }(\mathrm{kJ} \\
\left.\text { mol }^{-1}\right)\end{array}$ \\
\hline CA & -0.3847 & 0.350 & $2.76144 \mathrm{E}-01$ \\
\hline NA & 0.1640 & 0.325 & $7.11280 \mathrm{E}-01$ \\
\hline HÁ & 0.1934 & 0.250 & $1.25520 \mathrm{E}-01$ \\
\hline CS & -0.1111 & 0.350 & $2.76144 \mathrm{E}-01$ \\
\hline HS & 0.1251 & 0.250 & $1.25520 \mathrm{E}-01$ \\
\hline CW & 0.2318 & 0.350 & $2.76144 \mathrm{E}-01$ \\
\hline HW & 0.0398 & 0.250 & $1.25520 \mathrm{E}-01$ \\
\hline OY & -0.6547 & 0.307 & $7.11280 \mathrm{E}-01$ \\
\hline HY & 0.4537 & 0.000 & $0.00000 \mathrm{E}+00$ \\
\hline CG & 0.1650 & 0.350 & $2.76144 \mathrm{E}-01$ \\
\hline HG & 0.0600 & 0.250 & $1.25520 \mathrm{E}-01$ \\
\hline OG & -0.7200 & 0.300 & $7.11280 \mathrm{E}-01$ \\
\hline HO & 0.4350 & 0.000 & $0.00000 \mathrm{E}+00$ \\
\hline
\end{tabular}

Table S4. Bond and angle force constants, $\mathrm{k}_{\mathrm{r}}\left(\mathrm{kJ} \mathrm{mol}^{-1} \mathrm{~nm}^{-2}\right)$ and $\mathrm{k}_{\theta}\left(\mathrm{kJ} \mathrm{mol}^{-1} \mathrm{rad}^{-2}\right)$ and equilibrium distances and angles, $\mathrm{r}_{0}(\mathrm{~nm})$ and $\theta_{0}$ (degrees), used in FFM3 for choline $\left(\mathrm{Ch}^{+}\right)$.

\begin{tabular}{|c|c|c|c|c|c|}
\hline Bond & $\mathrm{r}_{0} / \mathrm{nm}$ & $\mathrm{k}_{\mathrm{r}}$ & Angle & $\mathrm{k}_{\theta}$ & $\theta_{0}$ \\
\hline HA-CA & 0.10990 & 142256.0 & HA-CA-HA & 146.44 & 110.01 \\
\hline CA-NA & 0.14980 & 205016.0 & HA-CA-NA & 146.44 & 108.90 \\
\hline NA-CS & 0.15160 & 205016.0 & CA-NA-CA & 230.12 & 108.73 \\
\hline CS-HS & 0.10805 & 142256.0 & CA-NA-CS & 216.73 & 110.20 \\
\hline CS-CW & 0.15210 & 132632.0 & NA-CS-HS & 146.44 & 106.40 \\
\hline CW-HW & 0.10850 & 142256.0 & NA-CS-CW & 292.88 & 116.60 \\
\hline CW-OY & 0.13950 & 188280.0 & HS-CS-HS & 146.44 & 108.60 \\
\hline OY-HY & 0.09490 & 231375.2 & HS-CS-CW & 146.44 & 109.30 \\
\hline & & & CS-CW-HW & 146.44 & 108.30 \\
\hline & & & CS-CW-OY & 334.72 & 109.60 \\
\hline & & & HW-CW-HW & 146.44 & 107.40 \\
\hline & & & HW-CW-OY & 146.44 & 111.60 \\
\hline & & & CW-OY-HY & 146.44 & 110.90 \\
\hline
\end{tabular}


Table S5. Bond and angle force constants, $\mathrm{k}_{\mathrm{r}}\left(\mathrm{kJ} \mathrm{mol}^{-1} \mathrm{~nm}^{-2}\right)$ and $\mathrm{k}_{\theta}\left(\mathrm{kJ} \mathrm{mol}^{-1} \mathrm{rad}^{-2}\right)$ and equilibrium distances and angles, $\mathrm{r}_{0}(\mathrm{~nm})$ and $\theta_{0}$ (degrees), used in FFM3 for ethylene glycol (EG).

\begin{tabular}{|c|c|c|c|c|c|}
\hline Bond & $\mathrm{r}_{0} / \mathrm{nm}$ & $\mathrm{k}_{\mathrm{r}}$ & Angle & $\mathrm{k}_{\theta}$ & $\theta_{0}$ \\
\hline CG-CG & 0.15290 & 224262.0 & CG-CG-OG & 418.40 & 108.00 \\
\hline CG-HG & 0.10900 & 284512.0 & CG-CG-HG & 313.80 & 110.70 \\
\hline CG-OG & 0.14300 & 267776.0 & HG-CG-HG & 276.14 & 107.80 \\
\hline OG-HO & 0.09450 & 462750.4 & HG-CG-OG & 292.88 & 109.50 \\
\hline \multicolumn{7}{r}{} & CG-OG-HO & 460.24 & 108.50 \\
\hline
\end{tabular}

Table S6. Dihedral parameters used in FFM3 for choline $\left(\mathrm{Ch}^{+}\right)$.

\begin{tabular}{|c|c|c|c|c|c|c|}
\hline Dihedral & $\mathrm{C}_{0}$ & $\mathrm{C}_{1}$ & $\mathrm{C}_{2}$ & $\mathrm{C}_{3}$ & $\mathrm{C}_{4}$ & $\mathrm{C}_{5}$ \\
\hline HA-CA-NA-CA & 1.7259 & 5.1777 & 0.0 & -6.9036 & 0.0 & 0.0 \\
\hline CA-NA-CS-HS & 5.6484 & 4.3932 & -4.1840 & -5.8576 & 0.0 & 0.0 \\
\hline CA-NA-CS-CW & 3.8702 & 3.8702 & -2.3012 & -5.4392 & 0.0 & 0.0 \\
\hline HA-CA-NA-CS & 1.9665 & 5.8994 & 0.0 & -7.8659 & 0.0 & 0.0 \\
\hline NA-CS-CW-HW & -40.271 & 17.259 & 29.288 & -6.2760 & 0.0 & 0.0 \\
\hline NA-CS-CW-OY & -26.778 & 32.635 & 20.920 & -26.778 & 0.0 & 0.0 \\
\hline HS-CS-CW-HW & 4.1840 & 0.0 & 12.552 & -16.736 & 0.0 & 0.0 \\
\hline HS-CS-CW-OY & -10.983 & 2.6150 & 10.460 & -2.0920 & 0.0 & 0.0 \\
\hline CS-CW-OY-HY & -0.7406 & 2.9414 & 0.7280 & -2.9288 & 0.0 & 0.0 \\
\hline HW-CW-OY-HY & -6.2760 & -6.2760 & -4.1840 & 16.736 & 0.0 & 0.0 \\
\hline
\end{tabular}

Table S7. Dihedral parameters used in FFM3 for ethylene glycol (EG).

\begin{tabular}{|c|c|c|c|c|c|c|}
\hline Dihedral & $\mathrm{C}_{0}$ & $\mathrm{C}_{1}$ & $\mathrm{C}_{2}$ & $\mathrm{C}_{3}$ & $\mathrm{C}_{4}$ & $\mathrm{C}_{5}$ \\
\hline OG-CG-CG-OG & 9.8520 & 11.989 & 4.9870 & -26.828 & 0.0 & 0.0 \\
\hline CG-CG-OG-OH & -0.1410 & 5.5910 & 3.1560 & -8.6060 & 0.0 & 0.0 \\
\hline HO-OG-CG-HG & 0.9414 & 2.8242 & 0.0000 & -3.7656 & 0.0 & 0.0 \\
\hline OG-CG-CG-HG & 0.9790 & 2.9372 & 0.0000 & -3.9162 & 0.0 & 0.0 \\
\hline HG-CG-CG-HG & 0.6276 & 1.8828 & 0.0000 & -2.5104 & 0.0 & 0.0 \\
\hline
\end{tabular}

\section{FFM4}

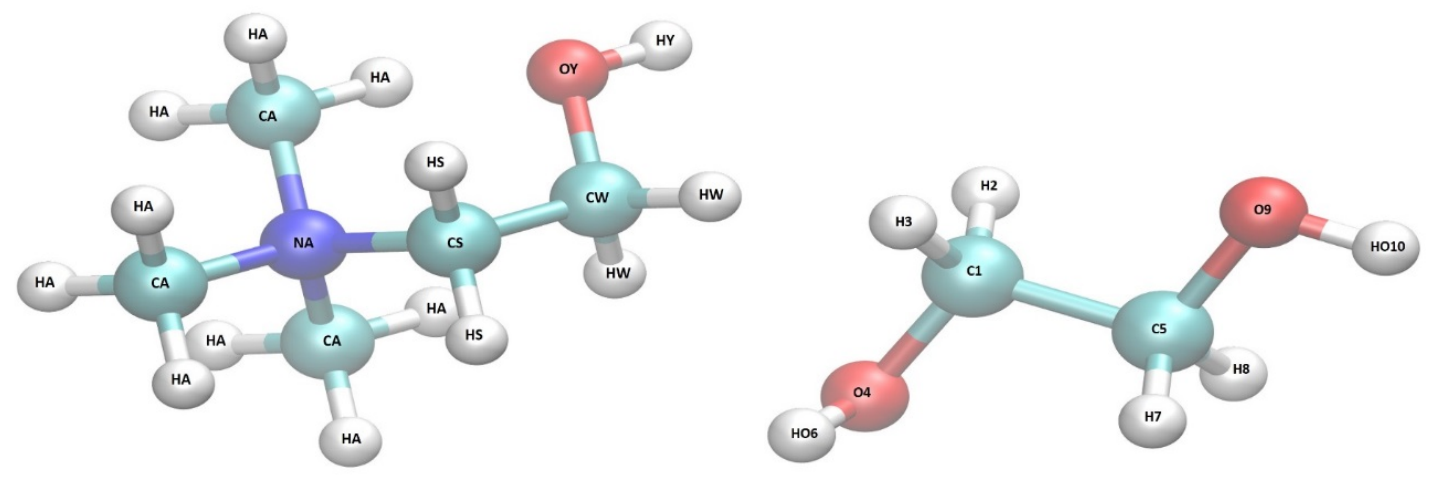

Figure S3. Nomenclature of atoms for choline $\left(\mathrm{Ch}^{+}\right)$and ethylene glycol (EG) for FFM4. 
$\mathbf{C h}^{+}$charges and parameters: J. Phys. Chem. B 117 (2013) 10250 - 10260;

Cl- parameters: J. Phys. Chem. B 108 (2004) 2038 - 2047

EG charges and parameters: J. Non-Crystalline Solids 364 ( 2013) 28 - 33

Table S8. Partial charges and nonbonded parameters used in FFM4 for choline $\left(\mathrm{Ch}^{+}\right)$, ethylene glycol (EG) and Chloride $\left(\mathrm{Cl}^{-}\right)$.

\begin{tabular}{|c|c|c|c|}
\hline Atom type & Partial charges for $\pm 1 \mathrm{e}$ & $\begin{array}{c}\text { vdW radius } \\
(\mathrm{nm})\end{array}$ & Well depth $\left(\mathrm{kJ} \mathrm{mol}^{-1}\right)$ \\
\hline CA & -0.1342 & 0.350 & $4.577296 \mathrm{E}-01$ \\
\hline NA & 0.0502 & 0.325 & $7.112800 \mathrm{E}-01$ \\
\hline HÁ & 0.1193 & 0.250 & $0.656888 \mathrm{E}-01$ \\
\hline CS & -0.0322 & 0.350 & $4.577296 \mathrm{E}-01$ \\
\hline HS & 0.1116 & 0.250 & $0.656888 \mathrm{E}-01$ \\
\hline CW & 0.1501 & 0.350 & $4.577296 \mathrm{E}-01$ \\
\hline HW & 0.0510 & 0.250 & $0.656888 \mathrm{E}-01$ \\
\hline OY & -0.6189 & 0.307 & $8.803136 \mathrm{E}-01$ \\
\hline HY & 0.4545 & 0.000 & $0.00000 \mathrm{E}+00$ \\
\hline C1 $=$ C5 & -0.001 & 0.358 & $-2.34304 \mathrm{E}-01$ \\
\hline H2 $=$ H7 & 0.137 & 0.239 & $-1.17152 \mathrm{E}-01$ \\
\hline H3 $=$ H8 & 0.156 & 0.239 & $-1.17152 \mathrm{E}-01$ \\
\hline O4 $=$ O9 & -0.733 & 0.315 & $-6.36386 \mathrm{E}-01$ \\
\hline HO6 $=$ HO10 & 0.441 & 0.040 & $-1.92464 \mathrm{E}-01$ \\
\hline Cl & -1.000 & 0.377 & $6.20000 \mathrm{E}-01$ \\
\hline
\end{tabular}

Table S9. Bond and angle force constants, $\mathrm{k}_{\mathrm{r}}\left(\mathrm{kJ} \mathrm{mol}^{-1} \mathrm{~nm}^{-2}\right)$ and $\mathrm{k}_{\theta}\left(\mathrm{kJ} \mathrm{mol}^{-1} \mathrm{rad}^{-2}\right)$ and equilibrium distances and angles, $\mathrm{r}_{0}(\mathrm{~nm})$ and $\theta_{0}$ (degrees), used in FFM4 for choline $\left(\mathrm{Ch}^{+}\right)$.

\begin{tabular}{|c|c|c|c|c|c|}
\hline Bond & $\mathrm{r}_{0} / \mathrm{nm}$ & $\mathrm{k}_{\mathrm{r}}$ & Angle & $\mathrm{k}_{\theta}$ & $\theta_{0}$ \\
\hline HA-CA & 0.109 & 283424.2 & HA-CA-HA & 326.6867 & 110.7 \\
\hline CA-NA & 0.150 & 245684.5 & HA-CA-NA & 410.1994 & 107.9 \\
\hline NA-CS & 0.150 & 245684.5 & CA-NA-CA & 525.8451 & 110.6 \\
\hline CS-HS & 0.109 & 283424.2 & CA-NA-CS & 525.8451 & 110.6 \\
\hline CS-CW & 0.154 & 253634.1 & NA-CS-HS & 410.1994 & 107.9 \\
\hline CW-HW & 0.109 & 281081.1 & NA-CS-CW & 539.3176 & 114.3 \\
\hline CW-OY & 0.143 & 262838.9 & HS-CS-HS & 327.8582 & 109.6 \\
\hline OY-HY & 0.097 & 309281.3 & HS-CS-CW & 385.0954 & 111.7 \\
\hline & & CS-CW-HW & 387.9405 & 110.1 \\
\cline { 4 - 6 } & & CS-CW-OY & 566.6810 & 109.4 \\
\cline { 4 - 6 } & & HW-CW-HW & 327.8582 & 109.6 \\
\cline { 4 - 6 } & & HW-CW-OY & 426.5170 & 109.9 \\
\cline { 4 - 6 } & & CW-OY-HY & 394.0491 & 108.2 \\
\cline { 4 - 6 }
\end{tabular}


Table S10. Bond and angle force constants, $\mathrm{k}_{\mathrm{r}}\left(\mathrm{kJ} \mathrm{mol}^{-1} \mathrm{~nm}^{-2}\right)$ and $\mathrm{k}_{\theta}\left(\mathrm{kJ} \mathrm{mol}^{-1} \mathrm{rad}^{-2}\right)$ and equilibrium distances and angles, $\mathrm{r}_{0}(\mathrm{~nm})$ and $\theta_{0}$ (degrees), used in FFM4 for ethylene glycol (EG).

\begin{tabular}{|c|c|c|c|c|c|}
\hline Bond & $\mathrm{r}_{0} / \mathrm{nm}$ & $\mathrm{k}_{\mathrm{r}}$ & Angle & $\mathrm{k}_{\theta}$ & $\theta_{0}$ \\
\hline $\mathrm{C}-\mathrm{C}$ & 0.1538 & 186188.0 & C-C-O & 633.4576 & 110.10 \\
\hline $\mathrm{C}-\mathrm{H}$ & 0.1111 & 258571.2 & C-C-H & 221.7520 & 110.10 \\
\hline $\mathrm{C}-\mathrm{O}$ & 0.1420 & 358150.4 & C-O-HO & 481.1600 & 106.00 \\
\hline O-HO & 0.0960 & 456056.0 & H-C-H & 297.0640 & 109.00 \\
\cline { 5 - 6 } & & O-C-H & 384.0912 & 108.89 \\
\cline { 5 - 6 }
\end{tabular}

Table S11. Dihedral parameters used in FFM4 for choline $\left(\mathrm{Ch}^{+}\right)$.

\begin{tabular}{|c|c|c|c|}
\hline Dihedral & $\mathrm{k}_{\phi} / \mathrm{kJ} \mathrm{mol}^{-1}$ & $\gamma /$ degrees & $\mathrm{n}$ \\
\hline HA-CA-NA-CA & 0.652704 & 0.00 & 3 \\
\hline CA-NA-CS-HS & 0.652704 & 0.00 & 3 \\
\hline CA-NA-CS-CW & 0.652704 & 0.00 & 3 \\
\hline HA-CA-NA-CS & 0.652704 & 0.00 & 3 \\
\hline NA-CS-CW-HW & 0.652704 & 0.00 & 3 \\
\hline NA-CS-CW-OY & 0.652704 & 0.00 & 3 \\
\hline HS-CS-CW-HW & 0.652704 & 0.00 & 3 \\
\hline HS-CS-CW-OY & 0.652704 & 0.00 & 3 \\
\hline CS-CW-OY-HY & 0.669440 & 0.00 & -3 \\
\hline HW-CW-OY-HY & 0.698728 & 0.00 & 3 \\
\hline
\end{tabular}

Table S12. Dihedral parameters used in FFM4 for ethylene glycol (EG).

\begin{tabular}{|c|c|c|c|}
\hline Dihedral & $\mathrm{k}_{\phi} / \mathrm{kJ} \mathrm{mol}^{-1}$ & $\gamma /$ degrees & $\mathrm{n}$ \\
\hline O-C-C-O & 0.79496 & 0.00 & 3 \\
\hline O-C-C-H & 0.79496 & 0.00 & 3 \\
\hline HO-O-C-C & 0.58576 & 0.00 & 3 \\
\hline HO-O-C-H & 0.58576 & 0.00 & 3 \\
\hline H-C-C-H & 0.79496 & 0.00 & 3 \\
\hline
\end{tabular}

\section{FFM5}

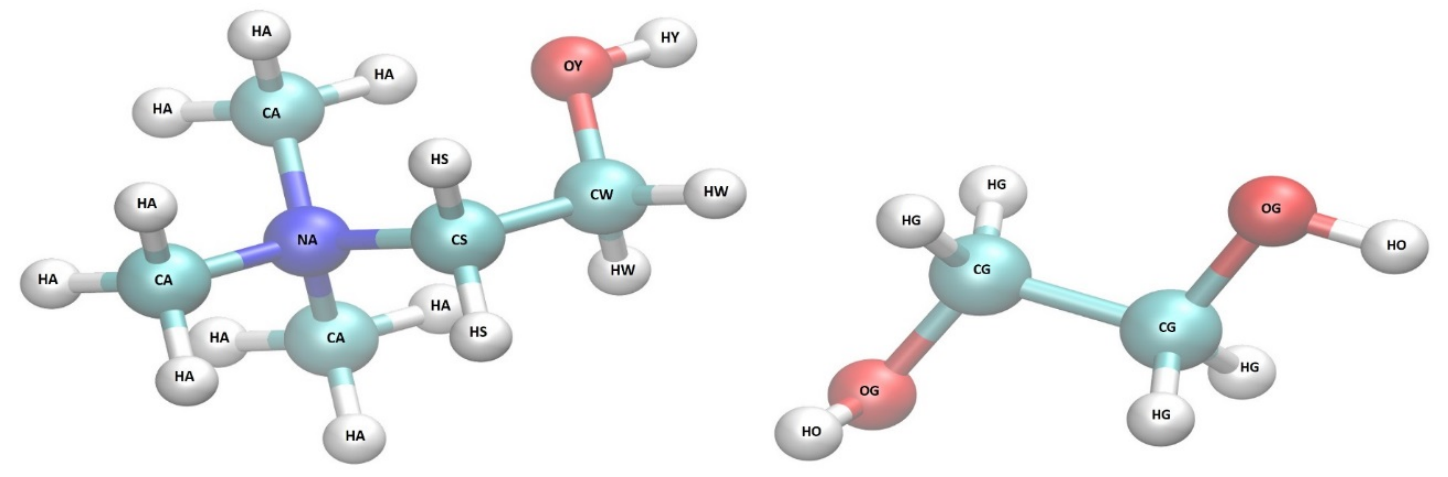

Figure S4. Nomenclature of atoms for choline $\left(\mathrm{Ch}^{+}\right)$and ethylene glycol (EG) for FFM5. 
$\mathbf{C h}^{+}$charges and parameters: J. Phys. Chem. B 117 (2013) 10250 - 10260;

Cl- parameters: J. Phys. Chem. B 108 (2004) 2038 - 2047

EG charges and parameters: J. Phys.Chem. B 115 ( 2011) 3013 - 3019

Table S13. Partial charges and nonbonded parameters used in FFM5 for choline $\left(\mathrm{Ch}^{+}\right)$, ethylene glycol (EG) and Chloride $\left(\mathrm{Cl}^{-}\right)$.

\begin{tabular}{|c|c|c|c|}
\hline Atom type & Partial charges for $\pm 1 \mathrm{e}$ & $\begin{array}{c}\text { vdW radius } \\
(\mathrm{nm})\end{array}$ & Well depth $\left(\mathrm{kJ} \mathrm{mol}^{-1}\right)$ \\
\hline CA & -0.1342 & 0.350 & $4.577296 \mathrm{E}-01$ \\
\hline NA & 0.0502 & 0.325 & $7.112800 \mathrm{E}-01$ \\
\hline HÁ & 0.1193 & 0.250 & $0.656888 \mathrm{E}-01$ \\
\hline CS & -0.0322 & 0.350 & $4.577296 \mathrm{E}-01$ \\
\hline HS & 0.1116 & 0.250 & $0.656888 \mathrm{E}-01$ \\
\hline CW & 0.1501 & 0.350 & $4.577296 \mathrm{E}-01$ \\
\hline HW & 0.0510 & 0.250 & $0.656888 \mathrm{E}-01$ \\
\hline OY & -0.6189 & 0.307 & $8.803136 \mathrm{E}-01$ \\
\hline HY & 0.4545 & 0.000 & $0.00000 \mathrm{E}+00$ \\
\hline CG & 0.1650 & 0.350 & $2.76144 \mathrm{E}-01$ \\
\hline HG & 0.0600 & 0.250 & $1.25520 \mathrm{E}-01$ \\
\hline OG & -0.7200 & 0.300 & $7.11280 \mathrm{E}-01$ \\
\hline HO & 0.4350 & 0.000 & $0.00000 \mathrm{E}+00$ \\
\hline Cl & -1.000 & 0.377 & $6.20000 \mathrm{E}-01$ \\
\hline
\end{tabular}

Table S14. Bond and angle force constants, $\mathrm{k}_{\mathrm{r}}\left(\mathrm{kJ} \mathrm{mol}^{-1} \mathrm{~nm}^{-2}\right)$ and $\mathrm{k}_{\theta}\left(\mathrm{kJ} \mathrm{mol}^{-1} \mathrm{rad}^{-2}\right)$ and equilibrium distances and angles, $\mathrm{r}_{0}(\mathrm{~nm})$ and $\theta_{0}$ (degrees), used in FFM5 for choline $\left(\mathrm{Ch}^{+}\right)$.

\begin{tabular}{|c|c|c|c|c|c|}
\hline Bond & $\mathrm{r}_{0} / \mathrm{nm}$ & $\mathrm{k}_{\mathrm{r}}$ & Angle & $\mathrm{k}_{\theta}$ & $\theta_{0}$ \\
\hline HA-CA & 0.109 & 283424.2 & HA-CA-HA & 326.6867 & 110.7 \\
\hline CA-NA & 0.150 & 245684.5 & HA-CA-NA & 410.1994 & 107.9 \\
\hline NA-CS & 0.150 & 245684.5 & CA-NA-CA & 525.8451 & 110.6 \\
\hline CS-HS & 0.109 & 283424.2 & CA-NA-CS & 525.8451 & 110.6 \\
\hline CS-CW & 0.154 & 253634.1 & NA-CS-HS & 410.1994 & 107.9 \\
\hline CW-HW & 0.109 & 281081.1 & NA-CS-CW & 539.3176 & 114.3 \\
\hline CW-OY & 0.143 & 262838.9 & HS-CS-HS & 327.8582 & 109.6 \\
\hline OY-HY & 0.097 & 309281.3 & HS-CS-CW & 385.0954 & 111.7 \\
\hline & & CS-CW-HW & 387.9405 & 110.1 \\
\cline { 4 - 6 } & & CS-CW-OY & 566.6810 & 109.4 \\
\cline { 4 - 6 } & & HW-CW-HW & 327.8582 & 109.6 \\
\cline { 4 - 6 } & & HW-CW-OY & 426.5170 & 109.9 \\
\cline { 4 - 6 } & & CW-OY-HY & 394.0491 & 108.2 \\
\cline { 4 - 6 }
\end{tabular}


Table S15. Bond and angle force constants, $\mathrm{k}_{\mathrm{r}}\left(\mathrm{kJ} \mathrm{mol}^{-1} \mathrm{~nm}^{-2}\right)$ and $\mathrm{k}_{\theta}\left(\mathrm{kJ} \mathrm{mol}^{-1} \mathrm{rad}^{-2}\right)$ and equilibrium distances and angles, $\mathrm{r}_{0}(\mathrm{~nm})$ and $\theta_{0}$ (degrees), used in FFM5 for ethylene glycol (EG).

\begin{tabular}{|c|c|c|c|c|c|}
\hline Bond & $\mathrm{r}_{0} / \mathrm{nm}$ & $\mathrm{k}_{\mathrm{r}}$ & Angle & $\mathrm{k}_{\theta}$ & $\theta_{0}$ \\
\hline CG-CG & 0.15290 & 224262.0 & CG-CG-OG & 418.40 & 108.00 \\
\hline CG-HG & 0.10900 & 284512.0 & CG-CG-HG & 313.80 & 110.70 \\
\hline CG-OG & 0.14300 & 267776.0 & HG-CG-HG & 276.14 & 107.80 \\
\hline OG-HO & 0.09450 & 462750.4 & HG-CG-OG & 292.88 & 109.50 \\
\hline \multicolumn{7}{r}{} & CG-OG-HO & 460.24 & 108.50 \\
\hline
\end{tabular}

Table S16. Dihedral parameters used in FFM5 for choline $\left(\mathrm{Ch}^{+}\right)$.

\begin{tabular}{|c|c|c|c|}
\hline Dihedral & $\mathrm{k}_{\phi} / \mathrm{kJ} \mathrm{mol}^{-1}$ & $\gamma /$ degrees & $\mathrm{n}$ \\
\hline HA-CA-NA-CA & 0.652704 & 0.00 & 3 \\
\hline CA-NA-CS-HS & 0.652704 & 0.00 & 3 \\
\hline CA-NA-CS-CW & 0.652704 & 0.00 & 3 \\
\hline HA-CA-NA-CS & 0.652704 & 0.00 & 3 \\
\hline NA-CS-CW-HW & 0.652704 & 0.00 & 3 \\
\hline NA-CS-CW-OY & 0.652704 & 0.00 & 3 \\
\hline HS-CS-CW-HW & 0.652704 & 0.00 & 3 \\
\hline HS-CS-CW-OY & 0.652704 & 0.00 & 3 \\
\hline CS-CW-OY-HY & 0.669440 & 0.00 & -3 \\
\hline HW-CW-OY-HY & 0.698728 & 0.00 & 3 \\
\hline
\end{tabular}

Table S1. Dihedral parameters used in FFM5 for ethylene glycol (EG).

\begin{tabular}{|c|c|c|c|c|c|c|}
\hline Dihedral & $\mathrm{C}_{0}$ & $\mathrm{C}_{1}$ & $\mathrm{C}_{2}$ & $\mathrm{C}_{3}$ & $\mathrm{C}_{4}$ & $\mathrm{C}_{5}$ \\
\hline OG-CG-CG-OG & 9.8520 & 11.989 & 4.9870 & -26.828 & 0.0 & 0.0 \\
\hline CG-CG-OG-OH & -0.1410 & 5.5910 & 3.1560 & -8.6060 & 0.0 & 0.0 \\
\hline HO-OG-CG-HG & 0.9414 & 2.8242 & 0.0000 & -3.7656 & 0.0 & 0.0 \\
\hline OG-CG-CG-HG & 0.9790 & 2.9372 & 0.0000 & -3.9162 & 0.0 & 0.0 \\
\hline HG-CG-CG-HG & 0.6276 & 1.8828 & 0.0000 & -2.5104 & 0.0 & 0.0 \\
\hline
\end{tabular}




\section{Auto-correlation functions}

\subsection{FFM1}

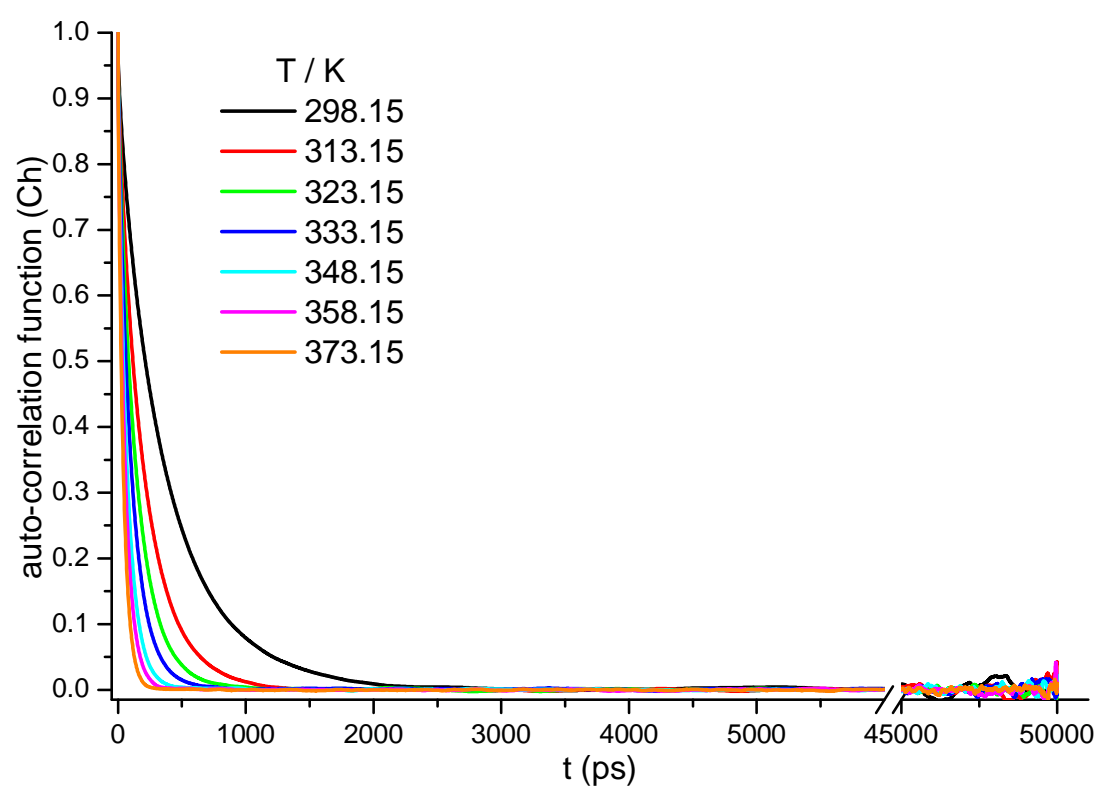

Figure S5. Time auto-correlation functions for choline $\left(\mathrm{Ch}^{+}\right)$obtained with 0.8FFM1.

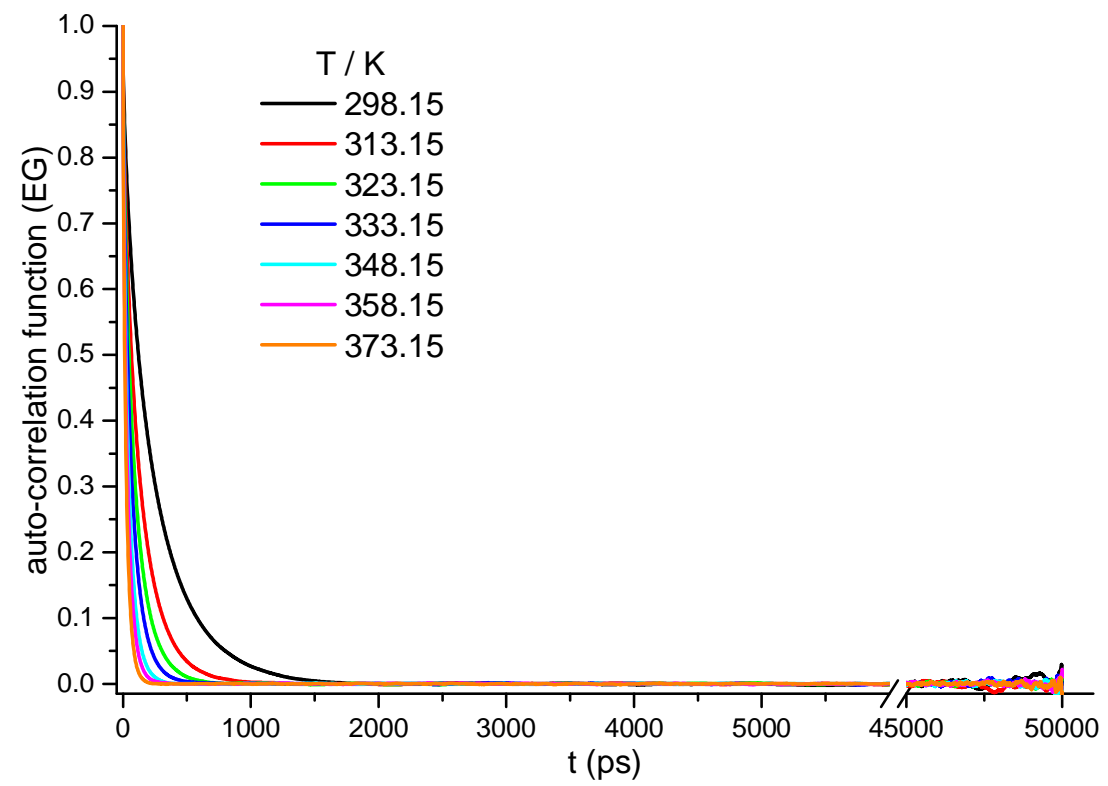

Figure S6. Time auto-correlation functions for ethylene glycol (EG) obtained with 0.8FFM1. 


\subsection{FFM2}

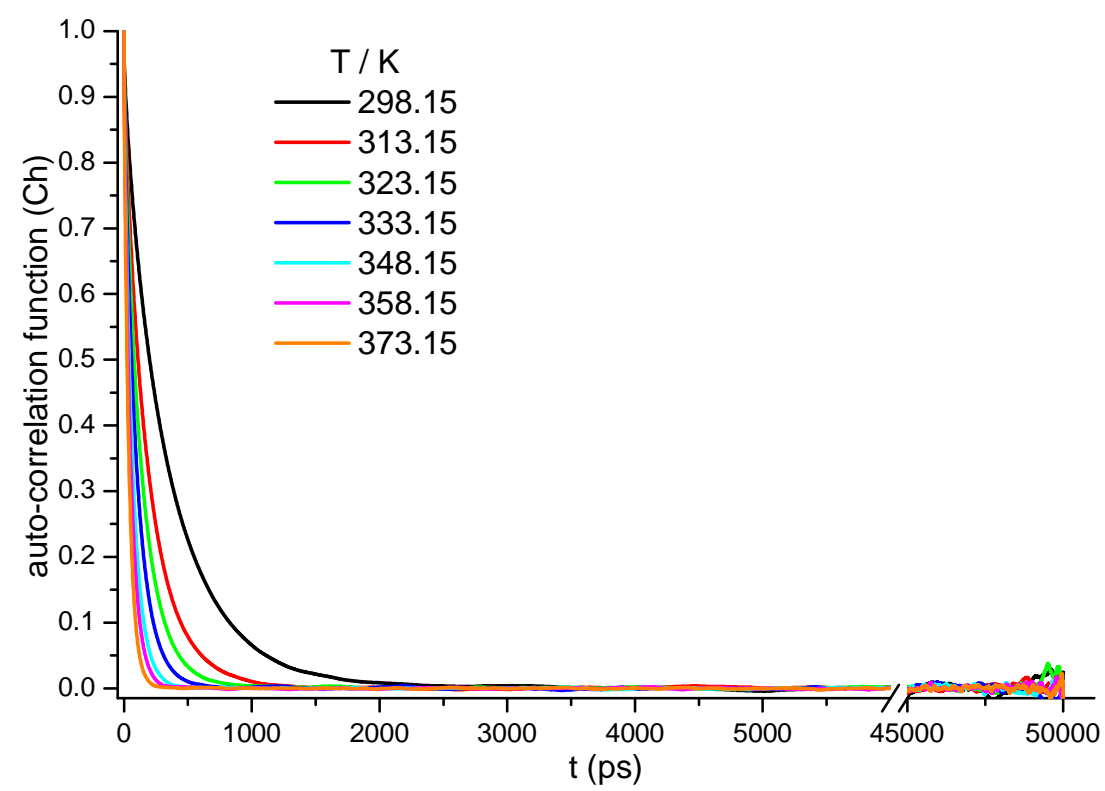

Figure S7. Time auto-correlation functions for choline $\left(\mathrm{Ch}^{+}\right)$obtained with 0.8FFM2.

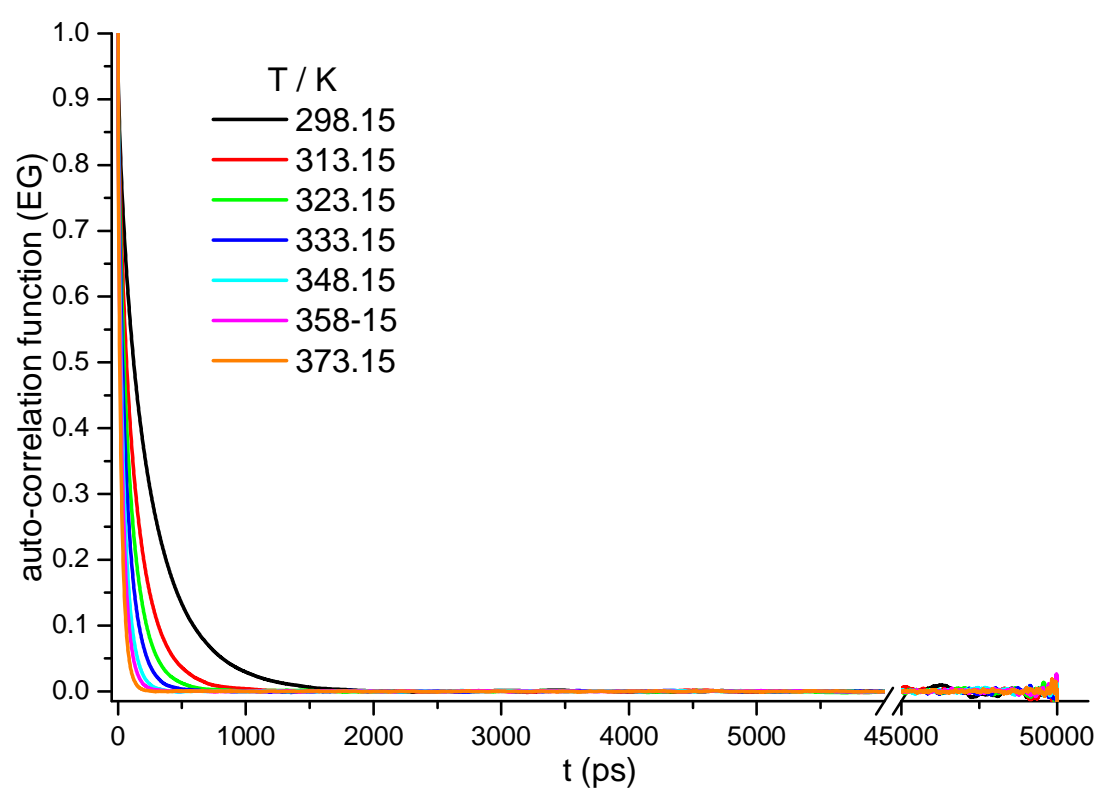

Figure S8. Time auto-correlation functions for ethylene glycol (EG) obtained with 0.8FFM2. 


\subsection{FFM3}

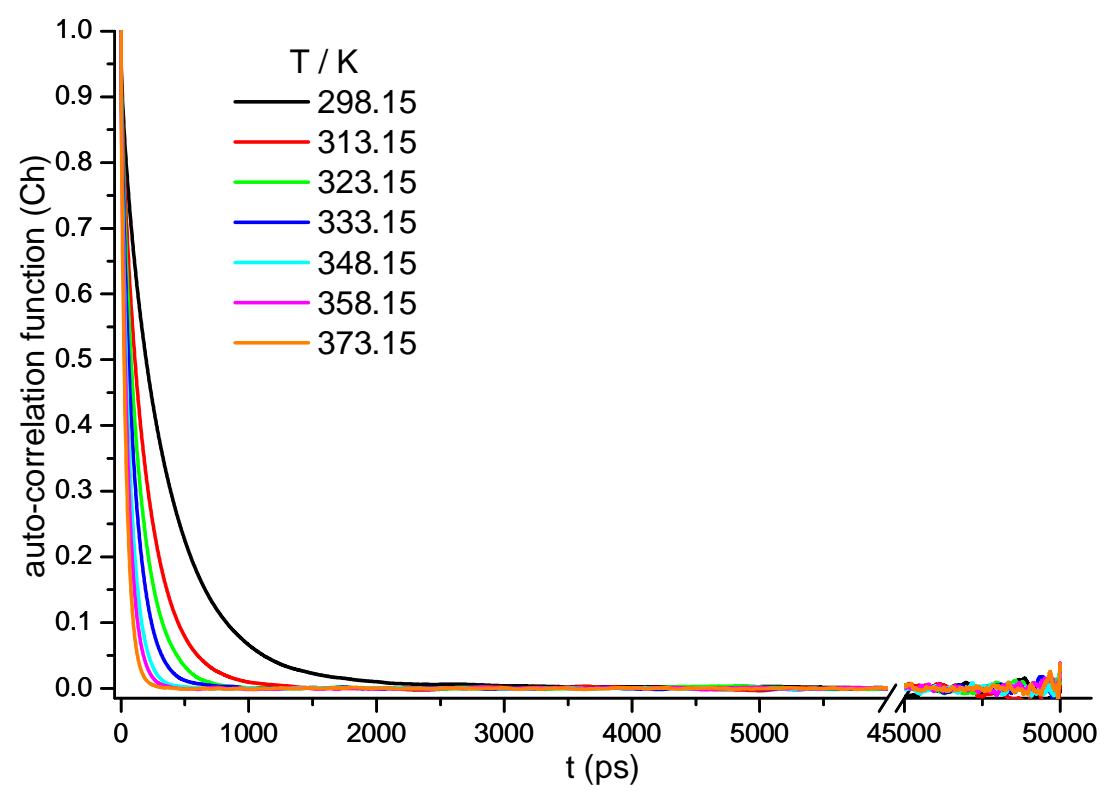

Figure S9. Time auto-correlation functions for choline $\left(\mathrm{Ch}^{+}\right)$obtained with $0.8 \mathrm{FFM} 3$.

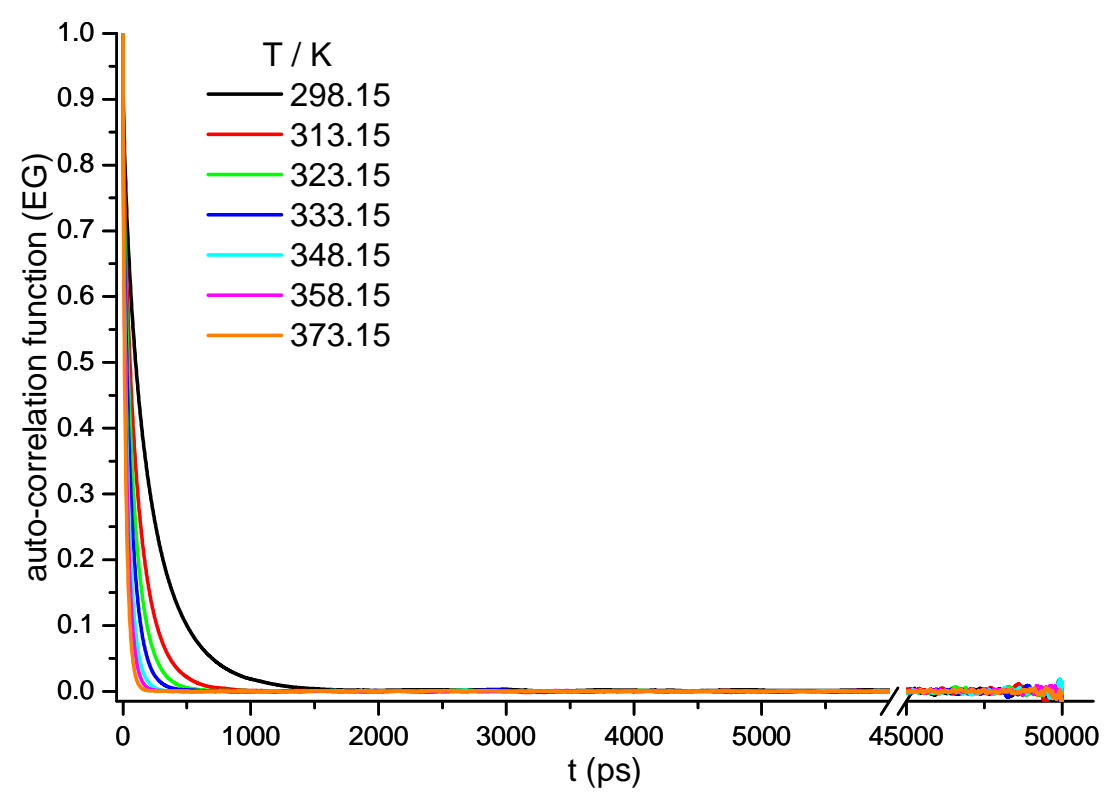

Figure S10. Time auto-correlation functions for ethylene glycol (EG) obtained with 0.8FFM3. 


\subsection{FFM4}

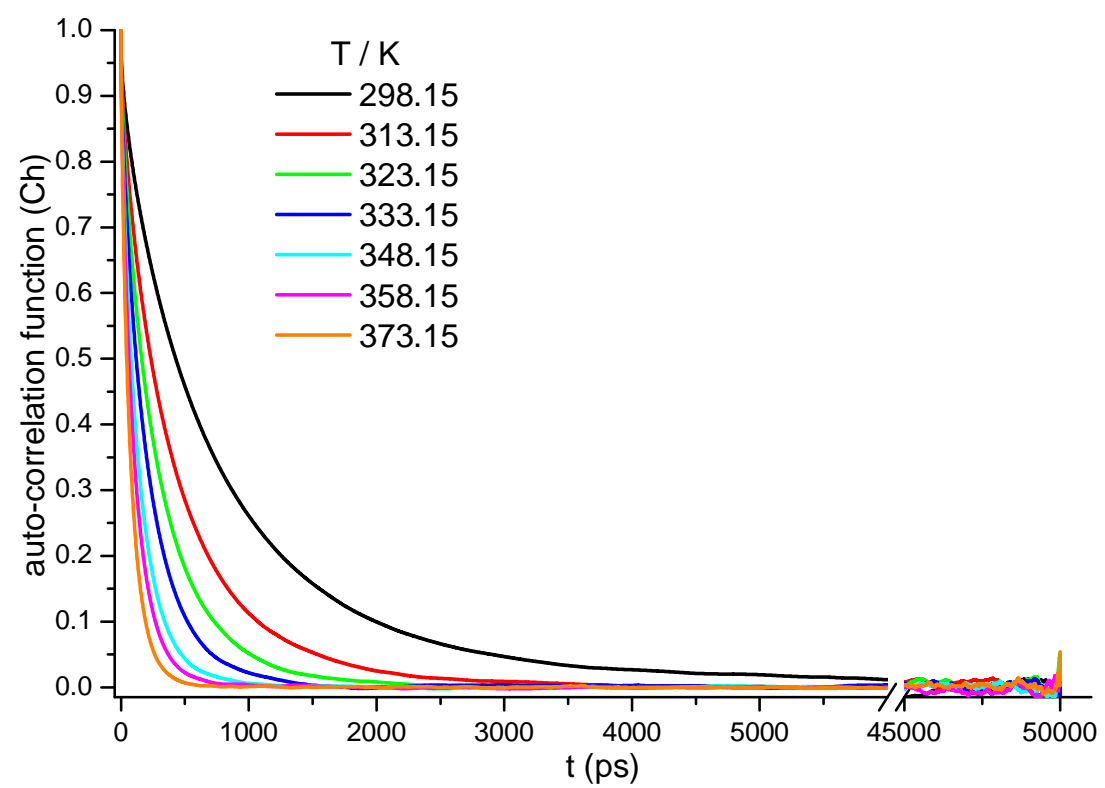

Figure S11. Time auto-correlation functions for choline $\left(\mathrm{Ch}^{+}\right)$obtained with 0.8FFM4.

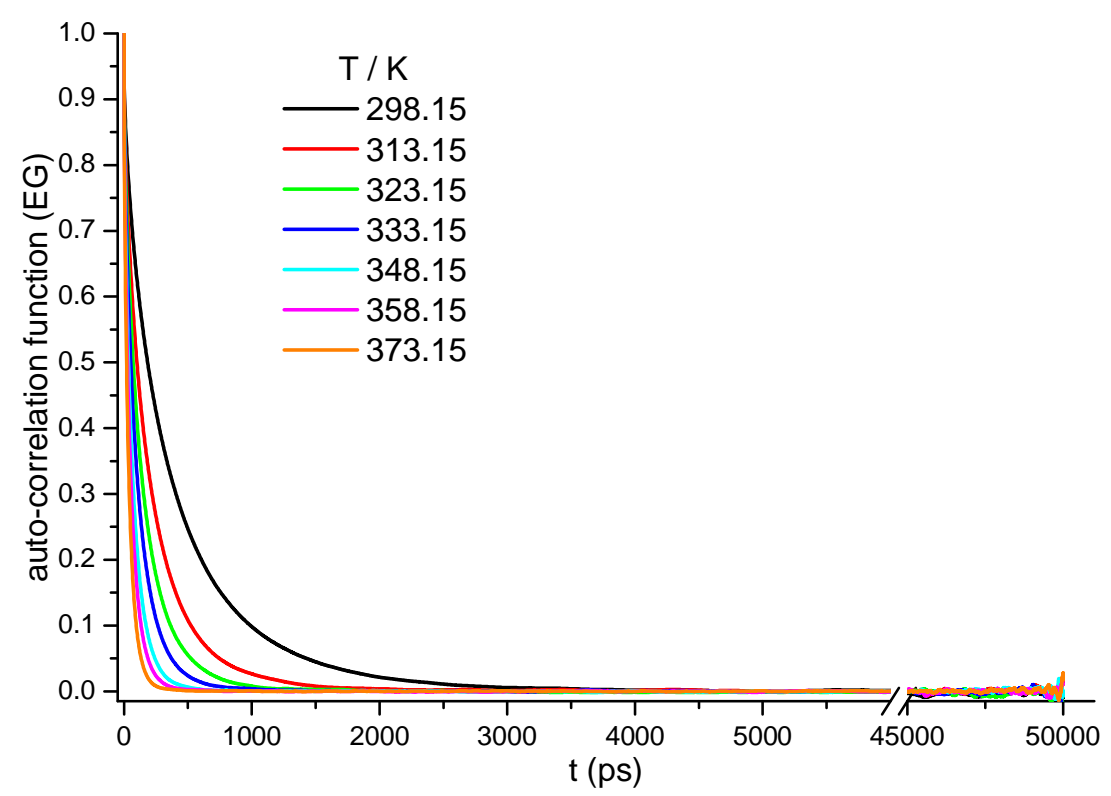

Figure S12. Time auto-correlation functions for ethylene glycol (EG) obtained with 0.8FFM4. 


\subsection{FFM5}

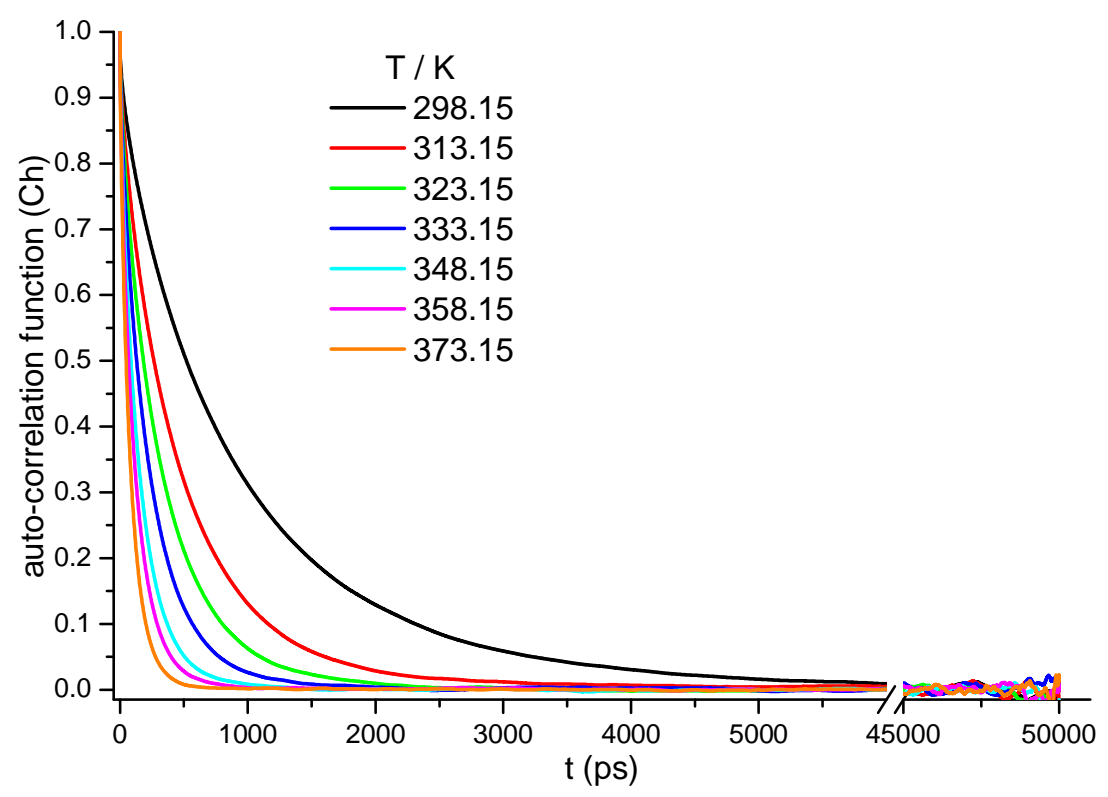

Figure S13. Time auto-correlation functions for choline $\left(\mathrm{Ch}^{+}\right)$obtained with 0.8FFM5.

\subsection{FFM5}

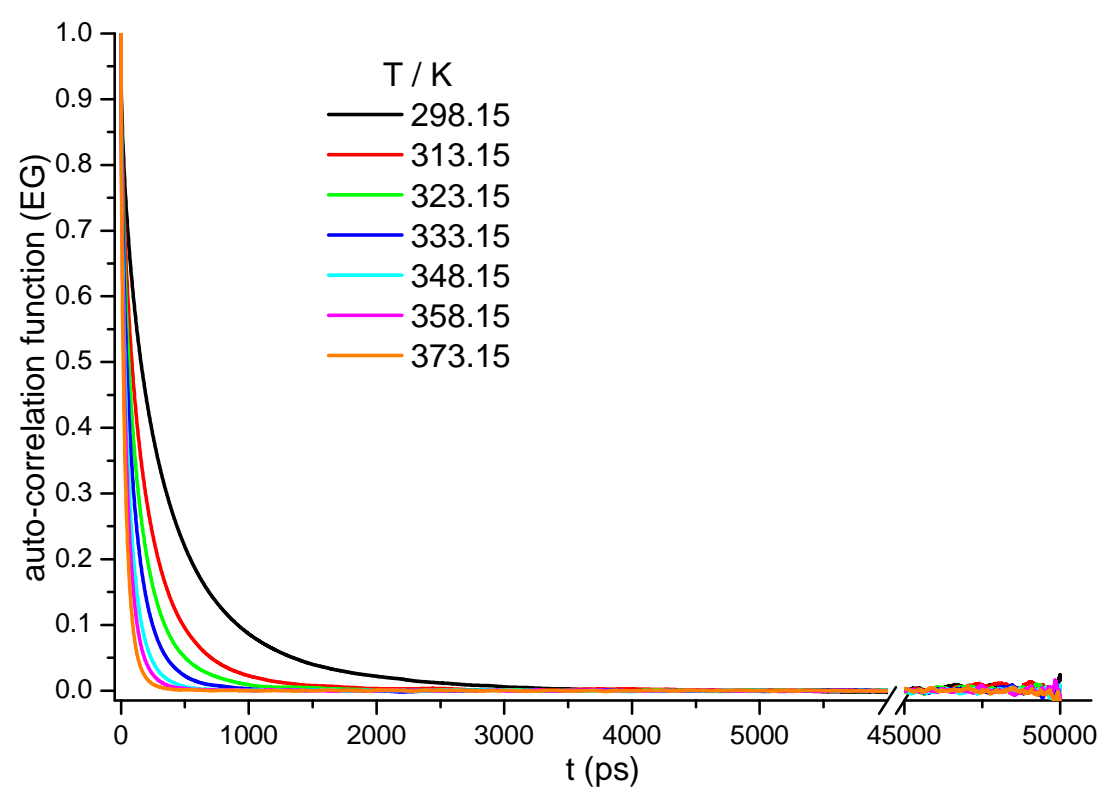

Figure S14. Time auto-correlation functions for ethylene glycol (EG) obtained with 0.8FFM5. 


\section{Ratios between self-diffusion coefficients}

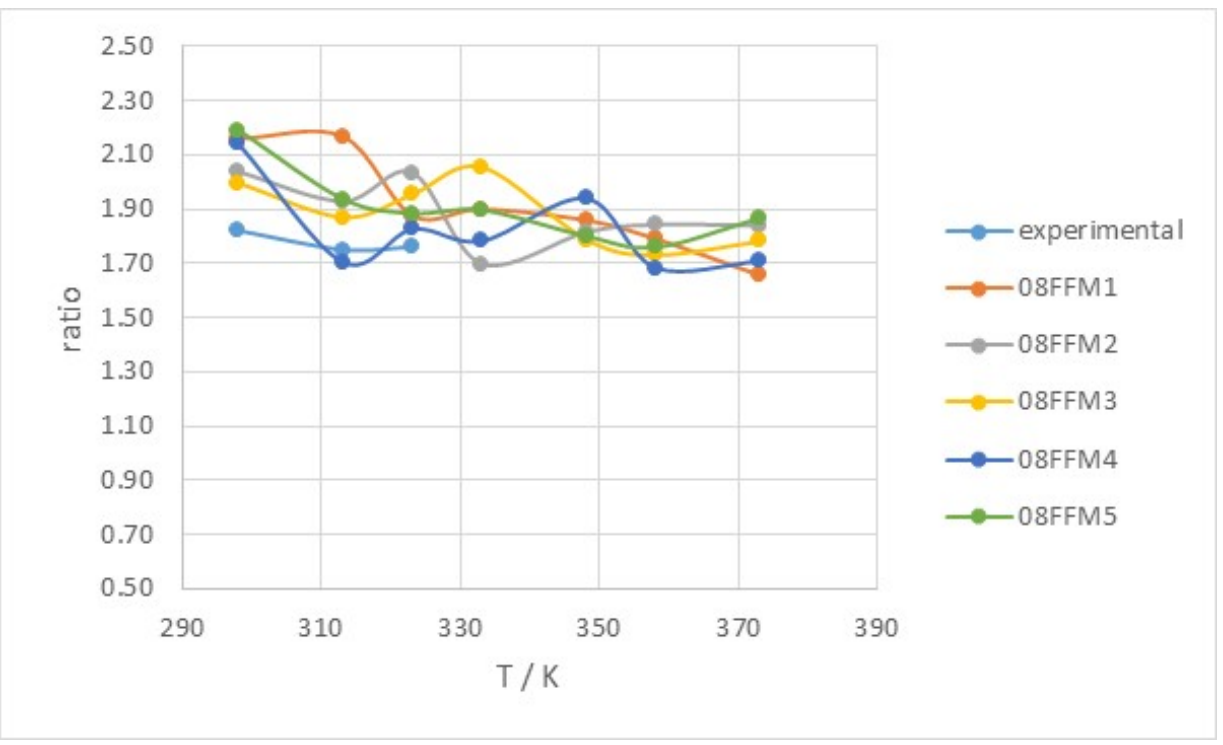

Figure S15. The ratio between the self-diffusion coefficients of HBD/cation

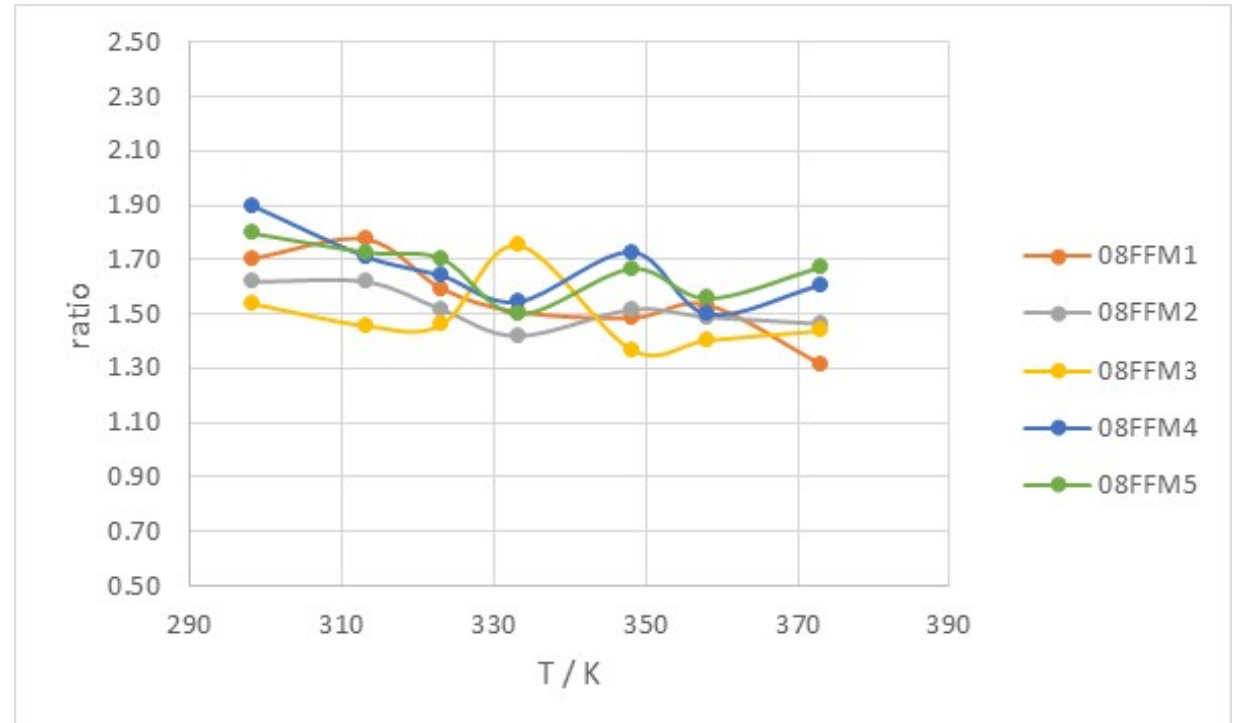

Figure S16. The ratio between the self-diffusion coefficients of anion/cation 


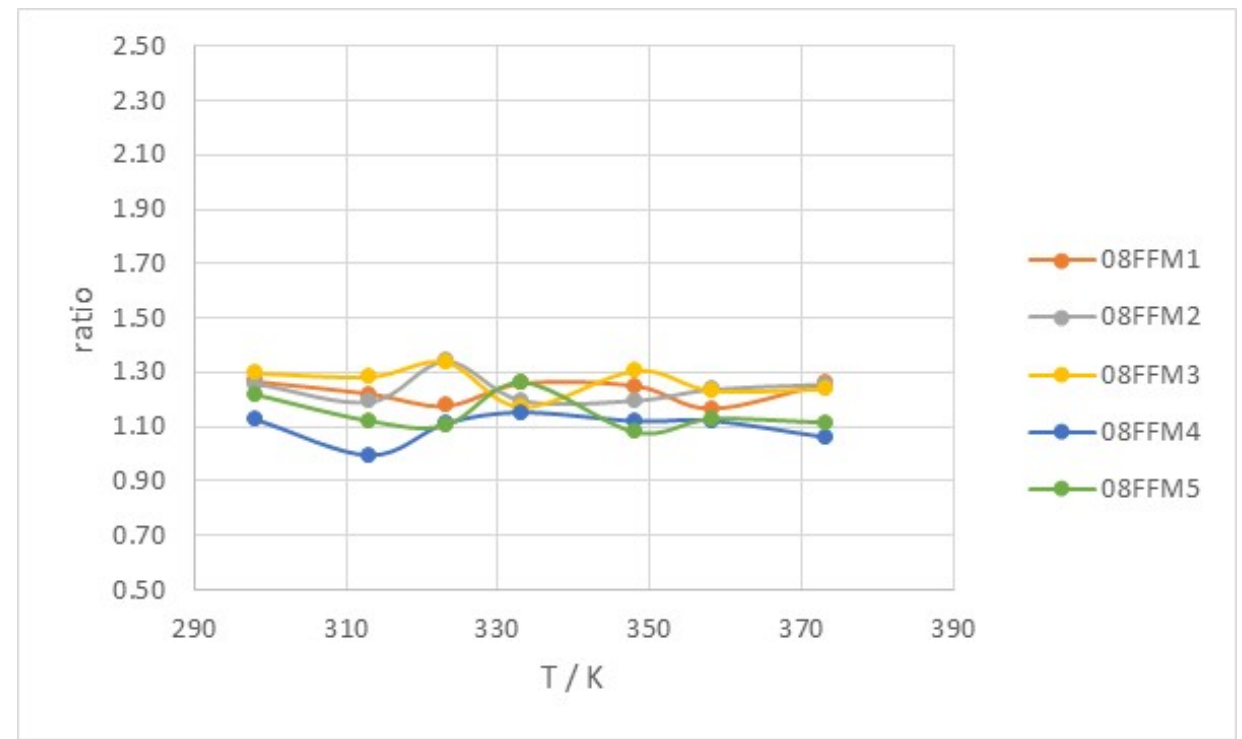

Figure S17. The ratio between the self-diffusion coefficients of HBD/anion

\section{RDFs}

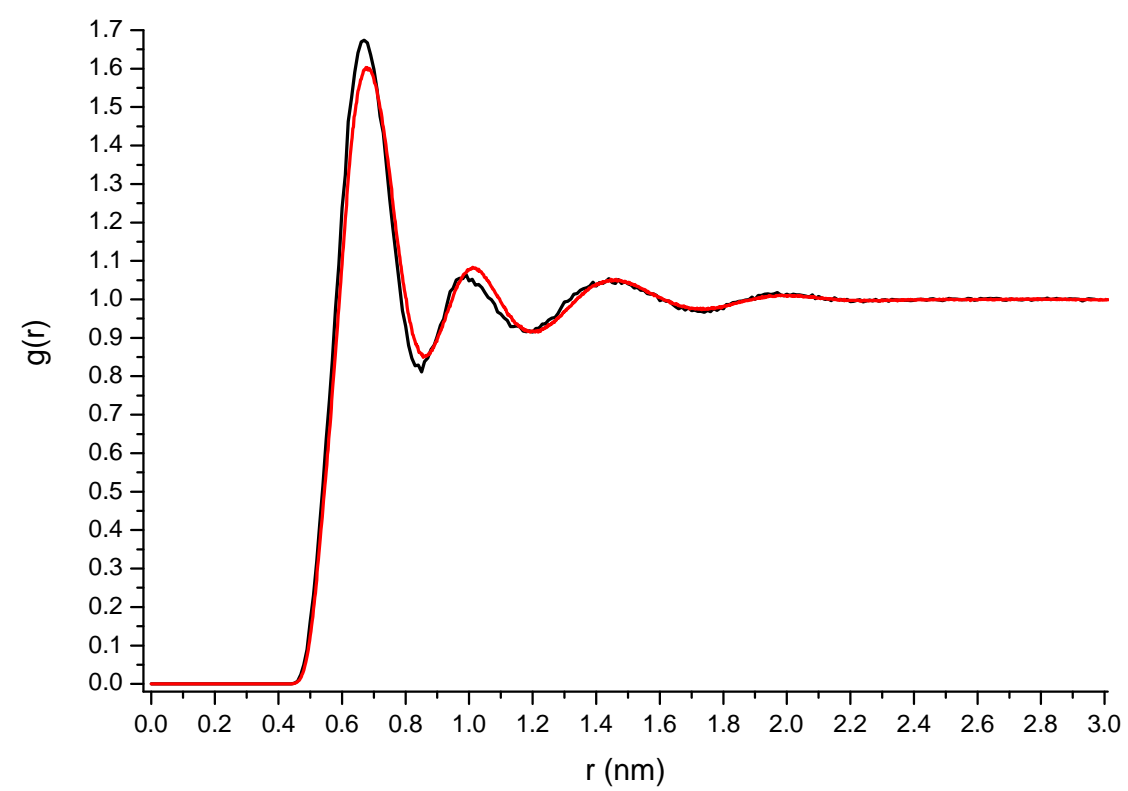

Figure S18. COM cation-cation RDF for ethaline, derived from MD simulations using FFM3 (black) and 0.8 FFM3 (red) at $373.15 \mathrm{~K}$. 


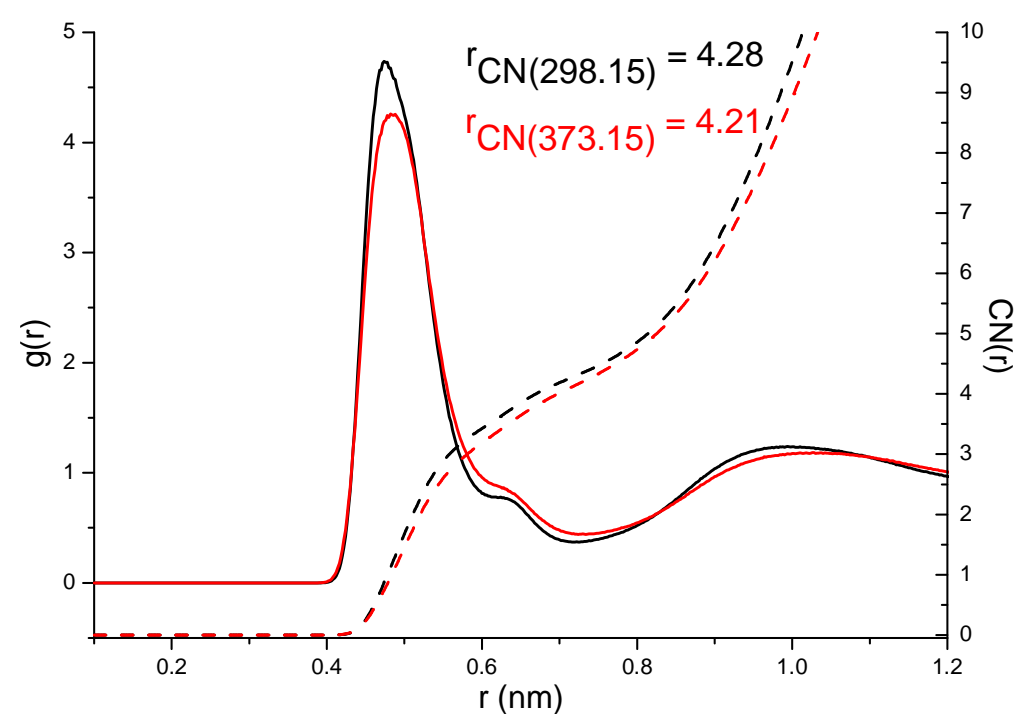

Figure S19. RDFs (solid lines) and CNs (dashed lines) for Cl-N, derived from MD simulations using 0.8FFM3 at $298.15 \mathrm{~K}$ (black) and $373.15 \mathrm{~K}$ (red).
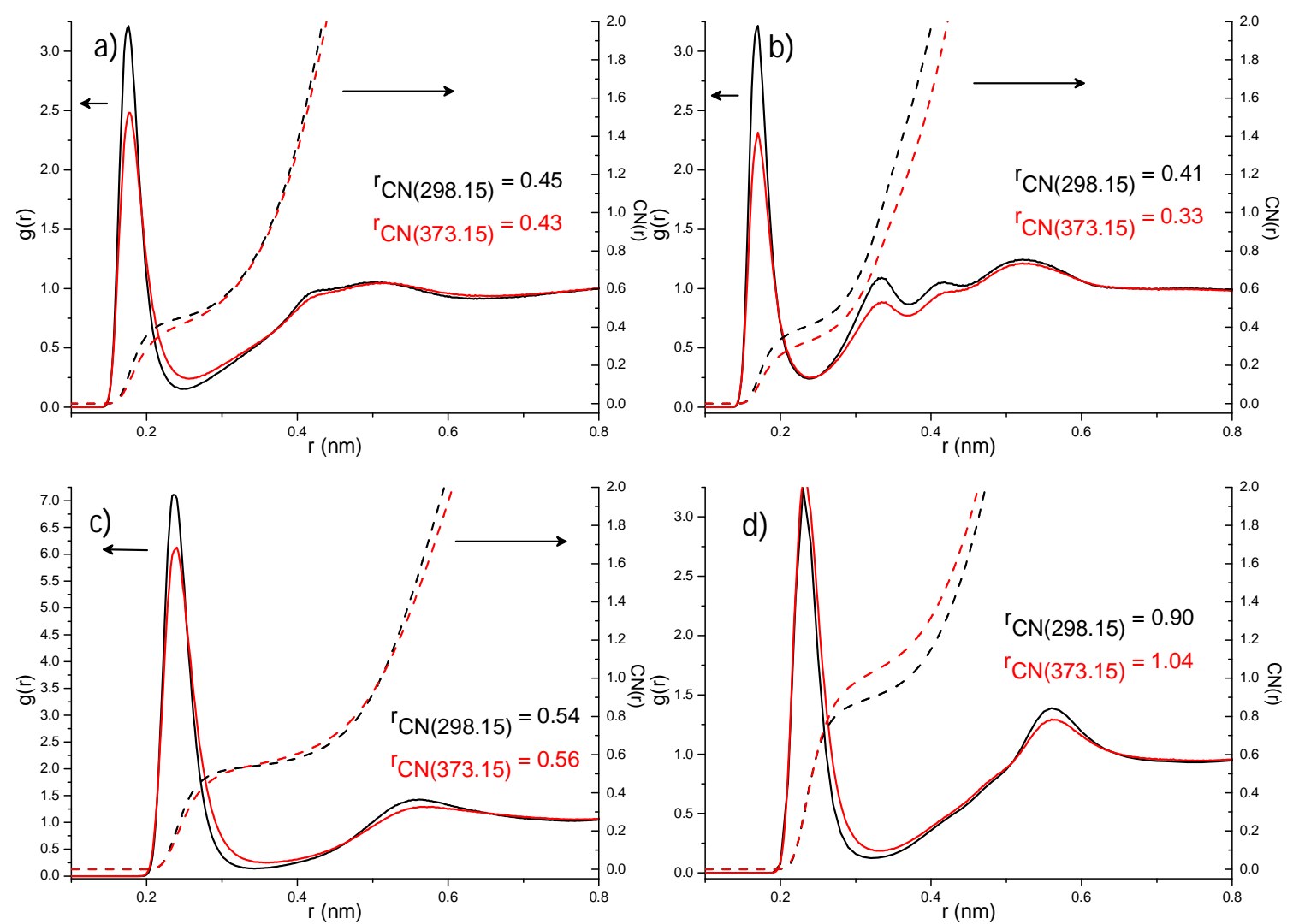

Figure S20. RDFs (solid lines) and running coordination numbers (dashed lines) for: (a) Og-Ho; (b) Og$\mathrm{Hg}$; (c) Cl-Ho and (d) Cl-Hg, at 298.15 K (black) and 373.25 K (red). 